Torsten Blunk ${ }^{1}$

Denis F. Hochstrasser ${ }^{2}$

Jean-Charles Sanchez ${ }^{2}$

Bernd W. Müller ${ }^{1}$

Rainer H. Mäller ${ }^{3}$

'Department of Pharmaceutics and Biopharmaceutics, University of Kiel ${ }^{2}$ Medicine Department and Medical Computing Center, Geneva University ${ }^{3}$ Department of Pharmaceutics and Biopharmaceutics, The Free

University of Berlin

\section{Colloidal carriers for intravenous drug targeting: Plasma protein adsorption patterns on surface-modified latex particles evaluated by two-dimensional polyacrylamide gel electrophoresis}

\begin{abstract}
Targeting to specific sites of the body via colloidal carriers is sought in order to reduce drug side effects. The adsorption of plasma proteins on intravenously injected particles is regarded as the key factor in explaining their organ distribution: total bound protein, or, more likely, the presence of specific proteins and their conformation, are expected to influence macrophage uptake. Polystyrene beads, $60 \mathrm{~nm}$ in diameter, were used as model carriers; their surface was differentially modified by adsorption of increasingly hydrophilic block copolymers, poloxamers 184,188 and 407 . After incubation in plasma, the patterns of protein adsorption onto coated beads were analyzed by highresolution two-dimensional polyacrylamide gel electrophoresis (2-D PAGE). The behavior of some representative proteins was monitored, including albumin, fibrinogen, IgG, factor B and the apolipoproteins, A-I, A-IV, C-III, E and $\mathrm{J}$. The more hydrophobic the particles, the larger the total amount of bound protein. However, this correlation was not valid for all of the analyzed protein species, which proves that it is insufficient to look only at physicochemical data to predict organ distribution. On the contrary, it is essential to use 2-D PAGE to establish the correlation between adsorbed proteins and carrier behavior in vivo.
\end{abstract}

\section{Introduction}

Colloidal carriers such as nanoparticles are a promising approach to drug targeting to specific organs of the body [1] in order to reduce side effects, e.g. in tumor therapy. The drug is incorporated in the polymeric nanoparticle which is composed of polymers that are biodegradable in the body (e.g. polylactide). After intravenous injection plasma proteins are immediately adsorbed onto the particles. These protein adsorption patterns are regarded as the key in their subsequent organ distribution. Depending on the particle surface properties, certain proteins will be preferentially adsorbed, leading to the adherence of the particle to cells with the appropriate receptor on the surface (Fig. 1). After attachment to the cell the nanoparticle is internalized by pinocytosis or phagocytosis and will release the drug inside the target cell. Carriers with different protein patterns will be recognized by different macrophage subpopulations ("differential adsorption", [2]). Carriers not recognized by macrophages circulate in the blood and can be used as circulating depots for the controlled release of drugs. The adsorption pattern acquired after intravenous injection depends on the physicochemical properties of the particles $[2,3]$. In vivo clearance of the particles by the mononuclear phagocytic system (MPS) was reported to increase with increasing hydrophobicity $[4,5]$ : hydrophobic particles were taken up by liver and spleen

Correspondence: Prof. Dr. Rainer H. Müller, Department of Pharmaceutics and Biopharmaceutics, University of Kiel, Gutenbergstr. 76-78, D-24118 Kiel, Germany

Abbreviations: 2-D, two-dimensional; HIC, hydrophobic interaction chromatography; PAGE, polyacrylamide gel electrophoresis; PDA, piperazine diacrylamide; SDS, sodium dodecyl sulfate macrophages within 5 min after injection in NZW rabbits (approximately $90 \%$ and $1-5 \%$ of the injected dose, respectively [1]). Controlled surface modification which reduces hydrophobicity decreases the uptake by the liver and spleen, and highly hydrophilic particles circulate in the blood. This correlation is only valid, however, if chemical groups which may lead to immunological recognition are absent (for details see [1]).

A simple but efficient method for carrier surface modification is the irreversible adsorption of hydrophilic block copolymers such as poloxamers (Fig. 2). They adsorb with their hydrophobic center part (poly(propylene oxide)) on the particle surface, while the hydrophilic poly(ethylene oxide) chains protrude into the dispersion medium, exposing a hydrophilic surface. The hydrophilicity increases with increasing molecular weight and length of the ethylene oxide chains of the poloxamer. This allows the controlled adjustment of surface properties. In addition, the nonionic adsorption layer covers the original charges on the particle surface, creating noncharged carriers in physiological salt solution [1], which is claimed to be an additional prerequisite for the reduction of liver and spleen uptake [6,7].

Despite the importance of the adsorption pattern for in vivo organ distribution and for successful drug targeting, up to now limited data is available, in general only from one-dimensional sodium dodecyl sulfate-polyacrylamide gel electrophoresis (SDS-PAGE) experiments [8]. To detect single proteins the adsorption patterns on the drug carriers were analyzed using high resolution twodimensional (2-D PAGE) according to Hochstrasser [9]. To establish the correlation between particle properties, protein adsorption layer, and organ distribution, it is sensible to use standard particles as model carriers, 


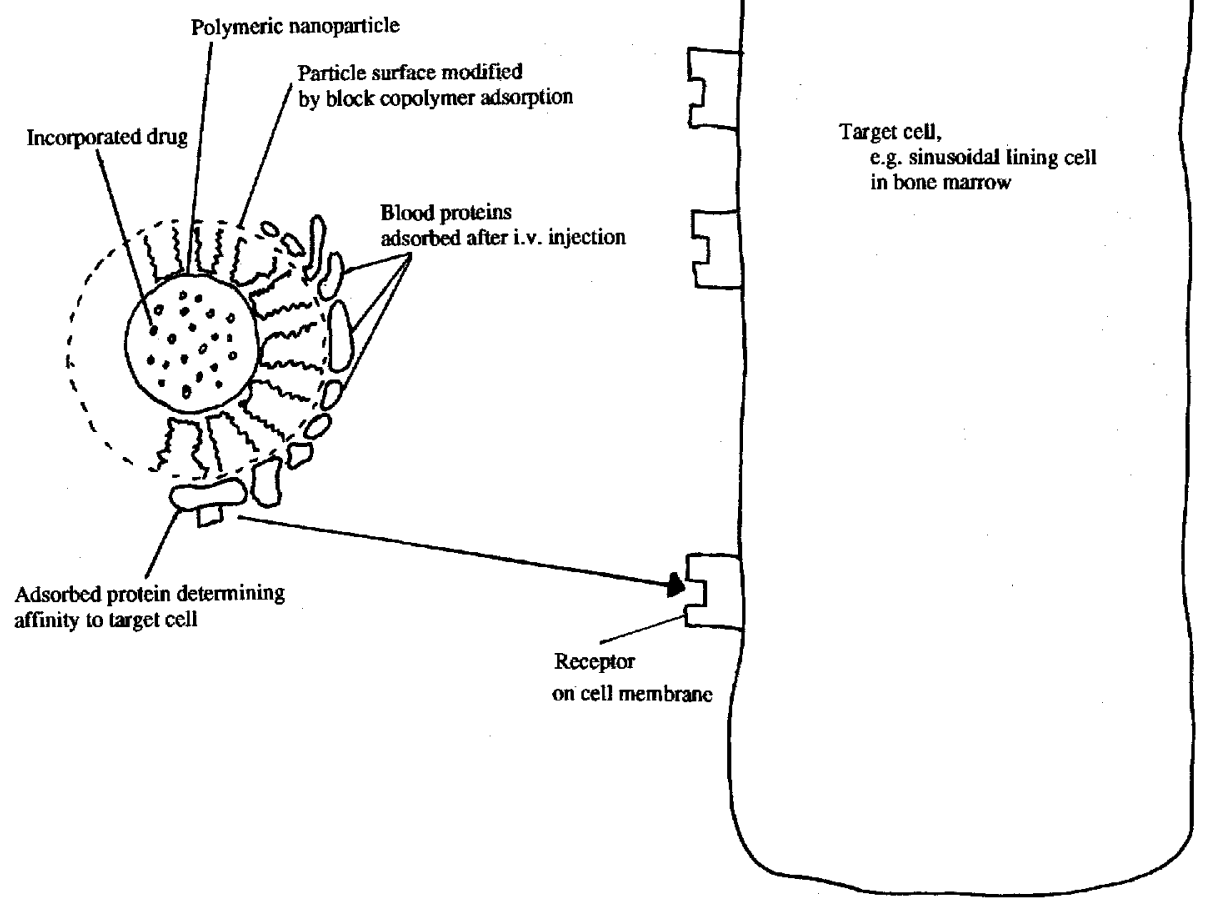

Figure 1. Principle of drug targeting using colloidal carriers for intravenous injection: The drug is incorporated inside the nanoparticle or alternatively in the capillaries of a sponge-like nanoparticle. The particle surface is modified by irreversible adsorption of block copolymers. Depending on the surface properties, certain blood proteins are preferentially adsorbed, leading to the adherence of the particle to cells with appropriate receptors. The particle hydrophobicity - and, subsequently, protein adsorption pattern and organ distribution - can be altered by using block copolymers of different molecular weight (see Fig. 2). which are well-defined in their surface properties and not biodegradable. The latter avoids superposition of effects of in vivo organ distribution and particle degradation by the body enzymes durin gamma scintigraphy studies. Polystyrene latex particles $(60 \mathrm{~nm})$, surfacemodified by coating with block copolymers poloxamer 184, 188 and 407 , were used as a model system (Fig. 2).

\section{Materials and methods}

\subsection{Reagents}

Polystyrene latex particles were from Polysciences (Warrington, USA). The block copolymers poloxamer 184, 188 and 407 were a gift from ICI (Manchester, UK). Human plasma was drawn from healthy male volunteers and stored at $-30^{\circ} \mathrm{C}$. For 2-D PAGE all chemicals were of analytical grade. Acrylamide, $N, N, N^{p}, N^{\prime}$-tetramethylethylenediamine (TEMED), ammonium persulfate and piperazine diacrylamide (PDA) were obtained from BioRad (Munich, Germany). Carrier ampholytes pH 3.5-10 and 4-8 were from BDH (Poole, UK), pH 9-11 from LKB (Bromma, Sweden). Cholamidopropyldimethylhydroxypropanesulfonate (CHAPS), Nonidet-P 40 and Tris were from Sigma (Deisenhofen, Germany). All other chemicals according to [9] were either from Fluka Chemie AG (Buchs, Switzerland) or Merck (Darmstadt, Germany).

\subsection{Apparatus}

The determination of particle size was performed by photon correlation spectroscopy using a Malvern spectro-

\section{Structure of poloxamer block copolymers}

$$
(\mathrm{EO})_{\mathrm{n}}-(\mathrm{PO})_{\mathrm{m}}-(\mathrm{EO})_{\mathrm{n}}
$$

Poloxamer $184: \mathrm{n}=13 ; \mathrm{m}=30 ; \mathrm{MW}=2900$

Poloxamer $188: \mathrm{n}=75 ; \mathrm{m}=30 ; \mathrm{MW}=8350$

Poloxamer $407: n=98 ; m=67 ; M W=11500$

\section{Surface modification of polystyrene drug carriers using poloxamers}

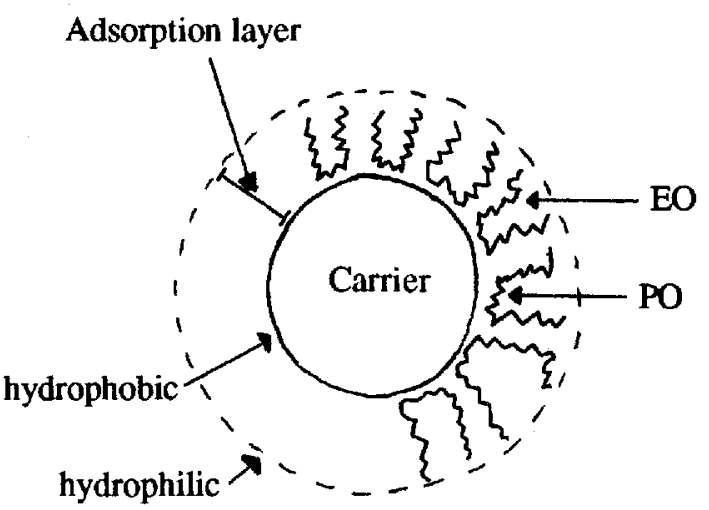

Figure 2. The poloxamer block copolymers adsorb on hydrophobic polystyrene drug carriers with their hydrophobic center part (poly(propylene oxide), $\mathrm{PO}$ ). The poly (ethylene oxide) (EO) chains protrude into the dispersion medium generating a hydrophilic surface. The hydrophilicity of the newly formed drug carrier surface increases with increasing ethylene oxide chain length and molecular weight of the poloxamers used. 
meter RR 102 in connection with a K 7025 correlator (Malvern Instruments, Malvern, UK). Isoelectric focusing using carrier ampholytes was carried out in a model 175 chamber from Bio-Rad (Munich, Germany). For SDS-PAGE the Bio-Rad Protean II chamber, the casting chamber $(160 \times 160 \times 1.5 \mathrm{~mm}$ gels $)$ and gradient pourer model 395 were used. Both the first- and seconddimensional separation were run with a Bio-Rad $1000 /$ 500 power supply. Silver-stained gels were scanned with a laser densitometer (Personal Densitometer from Molecular Dynamics, Krefeld, Germany). Data was processed on a SUN SPARCstation 10 using the MELANIE software [10].

\subsection{Sample preparation and 2-D PAGE}

Particle coating was carried out by mixing equal volumes of polystyrene (PS) latex particles $(2.5 \% \mathrm{w} / \mathrm{v})$ with poloxamer 184,188 or $407(2.0 \% \mathrm{w} / \mathrm{v}$, aqueous solution) and incubation overnight. Two hundred $\mu \mathrm{L}$ of these dispersions (PS-184, PS-188 and PS-407) were mixed with 5 $\mathrm{mL}$ human citrate plasma in a centrifugation tube and incubated for $5 \mathrm{~min}$ at $37^{\circ} \mathrm{C}$. The particle-to-plasma ratio was chosen to be relatively similar to the ratio used in the in vivo organ distribution studies $[1,11,12]$. In vivo $0.6 \mathrm{~mL}$ coated particles were injected in a $2-3 \mathrm{~kg} \mathrm{NZW}$ rabbit (blood volume $\sim 120 \mathrm{~mL}$ ). However, such low particle to plasma ratios were not used in this study because of the difficulties to remove such tiny amounts (60 $\mathrm{nm}$ nanoparticles) from large plasma quantities. The ratio of $200 \mu \mathrm{L}$ particle suspension to $5 \mathrm{~mL}$ plasma allows relatively easy separation with high particle yield even after multiple washing. After having established the basic method, future studies will include the variation of the particle-to-plasma ratio to assess any competitive phenomena of protein adsorption. For this purpose separation methods need to be established allowing the analysis of low particle concentrations in plasma. The particles were centrifuged for $90 \mathrm{~min}$ at $15000 \mathrm{~g}$. The supernatant was discarded, the particle pellet redispersed in $1.5 \mathrm{~mL}$ distilled water and the suspension transferred into Eppendorf vials. After centrifugation and discarding the supernatant a second washing step using distilled water was performed. Future studies will also include phosphate buffered saline (PBS) and Ringer solution as washing media to study possible desorption effects during sample preparation. The pellet obtained was dispersed in a protein solubilizing solution containing SDS and dithioerythritol (DTE), and the sample was processed like standard plasma samples. 2-D PAGE was performed essentially as described previously [9]. Plasma $(0.3 \mu \mathrm{L})$ or $40 \mu \mathrm{L}$ of the particle suspension were separated on the first-dimensional gels using carrier ampholytes. After SDS-PAGE the gels were silver-stained [9]. For a detailed methodological assessment of the protein adsorption onto drug carriers and its analysis using 2-D PAGE refer to Blunk et al. (submitted for publication). That publication also deals with adsorption onto chemically different particles.

\section{Results and discussion}

Intensive physicochemical characterization of the surface-modified polystyrene particles was performed previ- ously $[1,13]$. The thickness of adsorbed layers increased from poloxamer 184 to 188 and $407(2.4,7.6$ and $12.0 \mathrm{~nm}$, respectively). This increase was correlated with a decrease in hydrophobicity as determined by hydrophobic interaction chromatography (HIC). The HIC results showed marked differences in terms of surface hydrophobicity. The most hydrophobic poloxamer 184coated particles could only be eluted from very hydrophilic columns (ethyl-agarose), whereas poloxamer 107coated particles eluted from hydrophobic columns (such as pentyl-agarose) proved to be the most hydrophilic. It is concluded that the EO chains of poloxamer 407 are sufficiently long to shield the hydrophobic regions in the adsorption layer. Quantitative parameters to express the hydrophobicity of the particles were the elution volume, the percentage eluted from a column with buffer, percentage washed off from the column with $0.1 \%$ Triton $\mathrm{X}-100$ solution, percentage irreversibly bound to a column and the ratio of the areas under the curves of the elution to the wash peak, calculated for each column. For example, for the poloxamer 407, 188 and 184 coated particles the elution volumes of $4.5 \mathrm{~mL}(=$ void volume of column) and $6.5 \mathrm{~mL}$ and no elution, respectively, were obtained from a propyl-agarose column (for details see [1].

The adsorption patterns of the three kinds of particles clearly differed from plasma bulk solution (Fig. 3). This

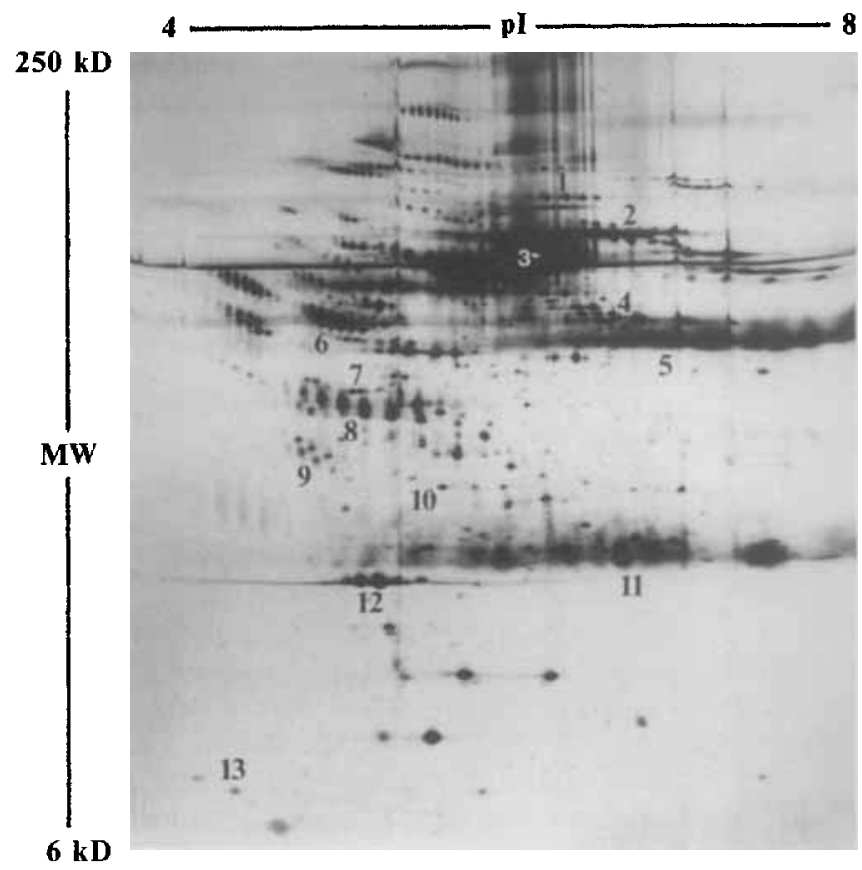

Figure 3. 2-D PAGE pattern of human plasma. The proteins exemplarily discussed in this paper are indicated: 1 , factor $B ; 2$, transferrin; 3 , albumin; 4 , fibronogen $\beta$ chain; 5, IgG $\gamma$ chain; 6 , $\alpha 1$-antitrypsin; 7, ApoA-IV; 8, haptoglobin $\beta$ chain; 9, ApoJ; 10 , ApoE; 11, Ig light chains; 12, ApoA-I; 13, ApoC-III. The analysis was performed essentially as described in [9]. First-dimensional isoelectric focusing was performed with carrier ampholytes, generating a $\mathrm{pH}$ gradient $4-8$. The polyacrylamide rods contained $4 \% \mathrm{~T}$ and $2.6 \% \mathrm{C}$ (PDA as cross-linker). Focusing was allowed to proceed for $52.3 \mathrm{Vh} / \mathrm{cm}^{2}$. Second-dimensional SDS-PAGE slab gels $(160 \times 160 \times 1.5 \mathrm{~mm})$ contained an $8-18 \% \mathrm{~T}$ linear acrylamide gradient with $2.6 \% \mathrm{C}$ (PDA as cross-linker) resolving proteins from 6 to $250 \mathrm{kDa}$. The gels were stained with silver according to [9]. 
observation is in agreement with data in the literature obtained using other analytical methods [3, 14]. Comparing the eluates from particles to plasma, the proportion of certain proteins is reduced or increased. The fraction of albumin and $\alpha 1$-antitrypsin (percentage of the total protein detected on the gel) is drastically reduced on the particles compared to plasma, and the hapto-
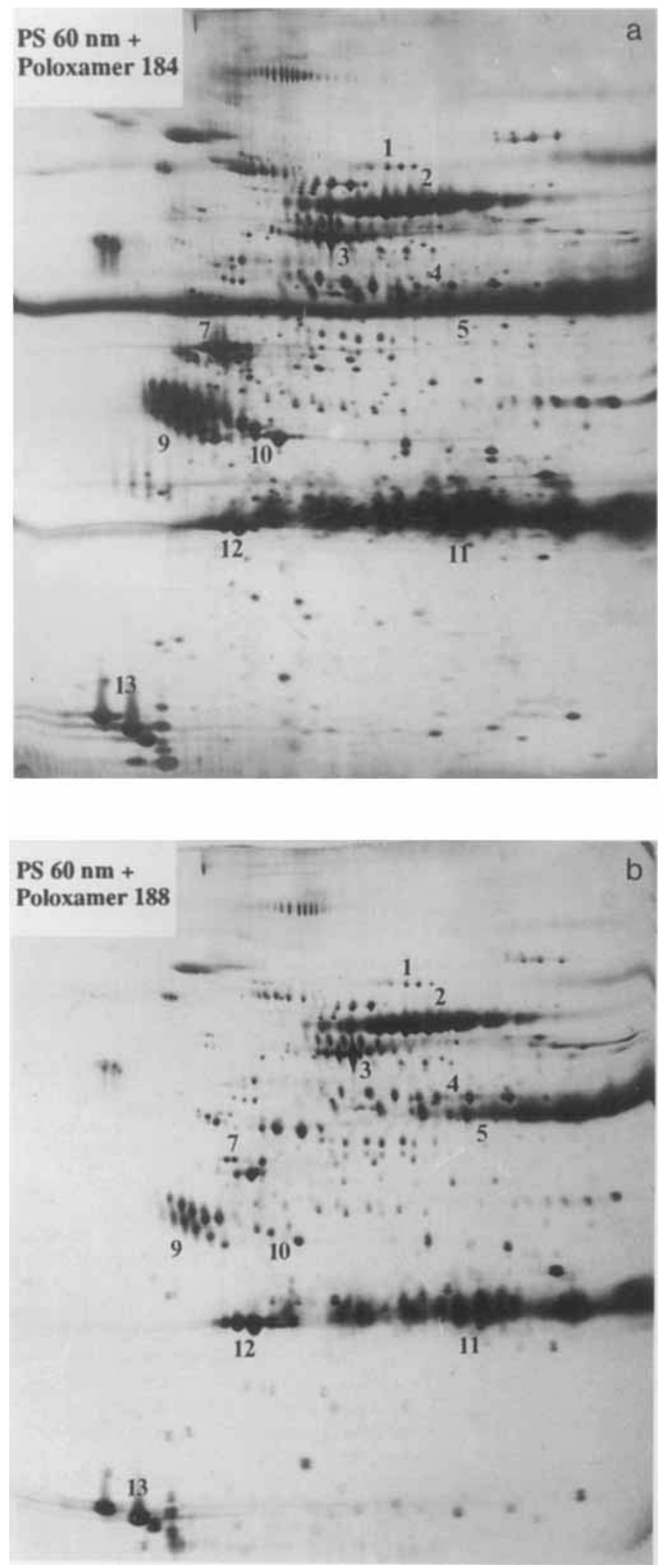

globin $\beta$ chain is hardly detectable. In contrast, transferrin and apolipoprotein C-III are examples for enriched proteins (Fig. $4 \mathrm{a}-\mathrm{c}$ ).

Comparing the three particle gels, differences were found in the total amount of bound protein as well as in the qualitative and quantitative composition of the

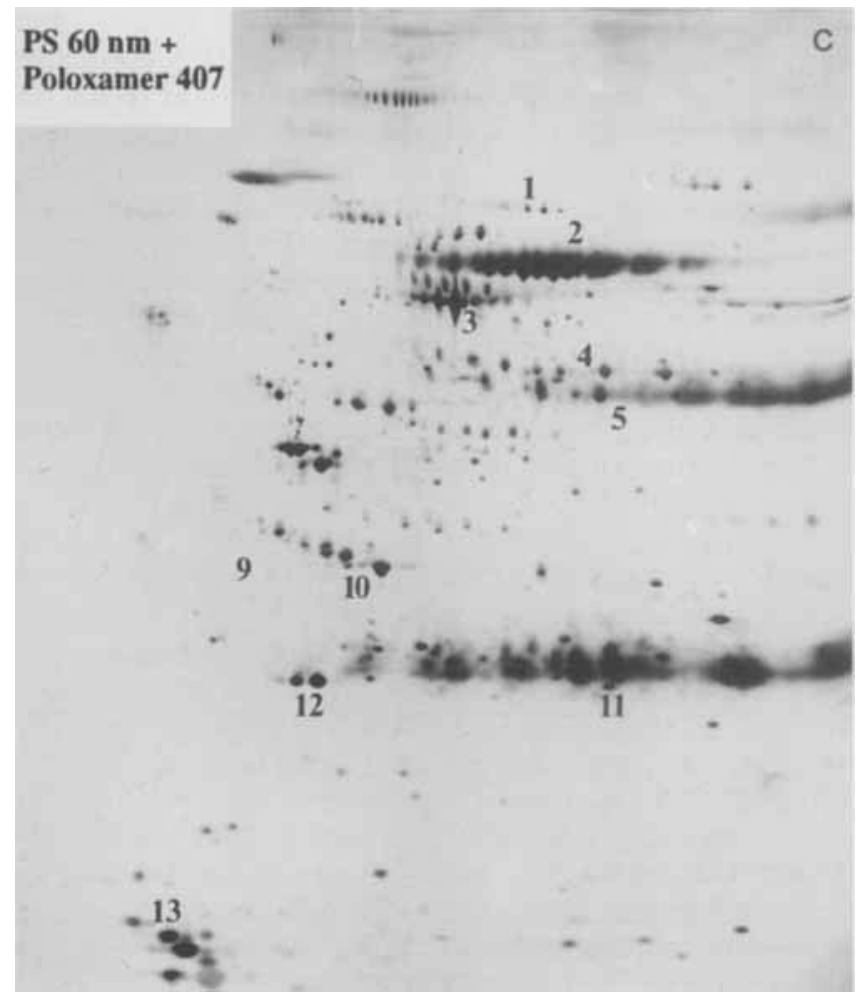

Figure 4. Plasma protein adsorption pattern of $60 \mathrm{~nm}$ polystyrene particles (PS) which were coated with poloxamer (a) 184, (b) 188 and (c) 407 . The numbers of the proteins indicated are the same as in Fig. 2. 
adsorption patterns. The differences in the total amount of protein adsorbed were assessed in a semi-quantitative manner on the basis of spot size and intensity of silver staining. At present a modified Lowry assay is about to be established to obtain absolute amounts of protein bound to differently modified particles. The total amount of adsorbed protein was found to be the largest on the most hydrophobic PS-184 $(970 \mathrm{cpm}$ units, determined using MELANIE software). The amount of adsorbed protein was distinctly lower on PS-188 (490 cpm units) and PS-407 (390 cpm units). This correlates with the physicochemical characterization by HIC. In order to exemplify the performance of the method it is now focused on single proteins. For most of them, again, the largest amount was detected on PS-184, IgG and transferrin being the major proteins. Only for apolipoproteins C-III and A-I similar amounts as on PS-188 were detected, for apo A-I the adsorption being even a little lower (Fig. 4 a-c, Table 1).

Comparing PS-188 to PS-407, transferrin, fibrinogen and factor B were found in similar amounts (Fig. $4 \mathrm{~b}-\mathrm{c}$,
Table 1). For apolipoproteins C-III, A-I and J the lowest binding was detected on the least hydrophobic PS-407, whereas apolipoprotein A-IV and E were adsorbed the least on PS-188 of intermediate hydrophobicity. Relatively larger fractions were found on PS-407 (Fig. 5).

The adsorption behavior of the latter apolipoproteins as well as of transferrin and apo C-III is therefore not correlated with surface hydrophobicity. This demonstrates that determination of surface hydrophobicity may be of limited use to predict the in vivo organ distribution of colloidal drug carriers. In contrast to reports in the literature $[4,5]$ a straigthforward correlation between surface hydrophobicity and macrophage uptake cannot be confirmed. Particle hydrophobicity is related to the total amount of protein adsorbed but provides no information about differential adsorption leading to differential macrophage uptake.

In general, using surface hydrophobicity as a parameter to differenciate between different particles with regard to their interaction with proteins is possible only for par-

Table 1: Examples of plasma proteins adsorbed on $60 \mathrm{~nm}$ polystrene (PS) model drug carriers, surface-modified with different poloxamers. Adsorption was detected by 2-D PAGE, numbers represent $\mathrm{cpm}$ units as a quantitative measure determined by MELANIE software.

\begin{tabular}{|c|c|c|c|c|c|c|c|}
\hline Model drug carrier & Factor B & Transferrin & Fibrinogen $\beta$ & Albumin & ApoC-III & $\begin{array}{c}\text { IgG heavy } \\
\text { chains }\end{array}$ & Ig light chains \\
\hline PS $60 \mathrm{~nm}+$ Poloxamer 184 & 3 & 69 & 18 & 20 & 28 & 73 & 141 \\
\hline PS $60 \mathrm{~nm}+$ Poloxamer 188 & 1 & 48 & 11 & 15 & 27 & 45 & 82 \\
\hline PS $60 \mathrm{~nm}$ + Poloxamer 407 & 1 & 44 & 8 & 10 & 7 & 34 & 78 \\
\hline
\end{tabular}

\begin{tabular}{lcccc}
\hline Model drug carrier & ApoA-I & ApoJ & ApoA-IV & ApoE \\
\hline PS 60 nm + Poloxamer 184 & 28 & 55 & 23 & 18 \\
PS 60 nm + Poloxamer 188 & 34 & 21 & 1 & 3 \\
PS 60 nm + Poloxamer 407 & 8 & 2 & 6 & 8
\end{tabular}

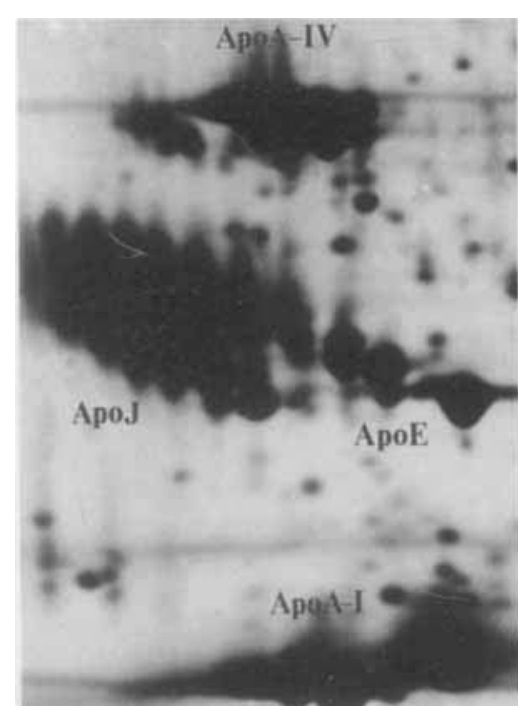

\section{PS $60 \mathrm{~nm}+$}

Poloxamer 184

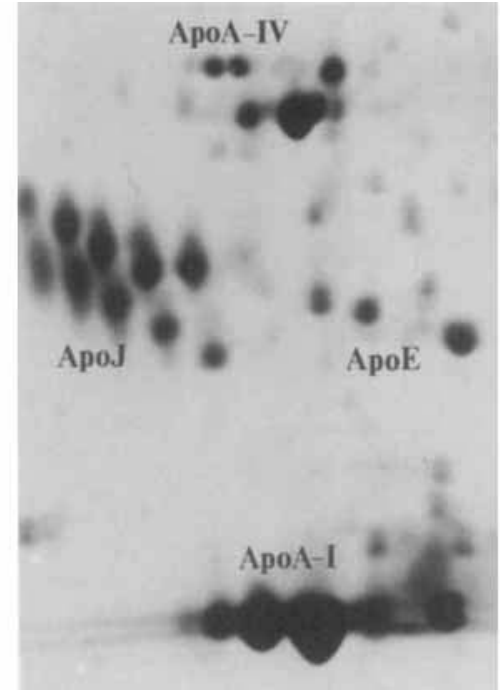

PS $60 \mathrm{~nm}+$

Poloxamer 188

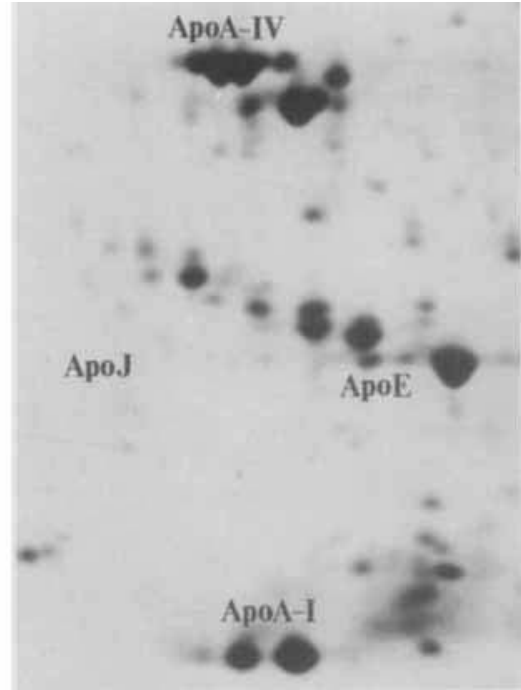

PS $60 \mathrm{~nm}+$

Poloxamer 407

Figure 5. Zoom of the middle left areas of the gels in Fig. 4: PS $60 \mathrm{~nm}+$ Poloxamer 184 (left), PS $60 \mathrm{~nm}+$ Poloxamer 188 (middle), PS $60 \mathrm{~nm}+$ Poloxamer 407 (right). For all apolipoproteins a latge amount was detected on PS-184, only for ApoA-I it was slightly smaller than on PS-188. ApoA-I and ApoJ were adsorbed the least on PS-407, Apo J being hardly detectable. In contrast, the smallest amounts of ApoA-IV and ApoE were observed on PS-188. 
ticles possessing a similar chemical composition on the surface. Surface chemistry strongly determines protein adsorption [15]. When comparing the adsorption patterns of poloxamer coated and uncoated particles, the differences between poly(ethylene oxide)/poly(propylene oxide) and polystyrene lead to qualitatively and quantitatively different results (Blunk et al., submitted for publication).

Differences in the adsorption patterns could be detected but their relevance to organ distribution needs to be assessed. At first sight, the heavy protein adsorption observed on the most hydrophobic poloxamer 184 coated particles seems to be in agreement with the in vivo liver uptake observed with other hydrophobic particles. The clearly reduced amount of adsorbed protein on more hydrophilic particles seems to correlate with reduction, or avoidance, of liver uptake by more hydrophilic particles [11]. However, not the total amount of protein but the composition of the protein layer is regarded as the important factor. For example, cell culture experiments indicate the existence of an opsonin which mediates specifically the uptake by liver macrophages and a second opsonin for the uptake by spleen macrophages [16-18]. Opsonization of erythrocytes with IgG led to preferential uptake by spleen macrophages and with $\operatorname{IgM}$ to preferential uptake by liver macrophages [19]. Proteins present in large amounts on the particles, e.g. IgG, are the most likely to affect organ distribution. There may also be a minimum concentration for certain proteins required to cause an effect, and proteins present in minute amounts are unlikely to be of importance. Furthermore, differences in the conformation of proteins possibly generated by different surface chemistry and/or neighboring proteins in the adsorption layer - may also be a determining factor for the fate of the carriers in vivo. The assumption that the composition of the layer (single proteins, conformation of proteins) is the main factor is supported by the organ distribution of poloxamine 908 and poloxamer 407 coated particles. They adsorb small amounts of total protein resulting in similar adsorption patterns, but differences are still detectable (Blunk et al., submitted for publication). Both particles are able to avoid liver/spleen uptake but the poloxamine 908 particles circulate in blood [11], whereas the poloxamer 407 coated particles accumulate in bone marrow [12].

\section{Concluding remarks}

High resolution 2-D PAGE was able to detect major differences in the adsorption patterns on particles surfacemodified with chemically similar block copolymers. The total amount of adsorbed protein was related to surface hydrophobicity, whereas quantitative differences for single proteins could not be linearly correlated to the differences in surface hydrophobicity. Therefore analysis of the adsorption patterns appears to be a straightforward and more sensible approach to understand differences in organ distribution than studies solely based on physicochemical characterization. In the near future further data processing will be performed using the MELANIE software [16]. Nevertheless, a physicochemical characteriza- tion of the particles is needed to complement 2-D PAGE analysis, especially when comparing particles similar in the chemical composition of the surface.

The final goal will be to establish the correlation between physicochemical particle properties, acquired protein adsorption after intravenous injection and the resulting organ distribution. Having established this correlation, particles can be targeted by controlled adjustment of their surface properties. After intravenous injection they will adsorb the protein species which are responsible for the accumulation in the desired target tissue, e.g. bone marrow. In comparison to homing via antibodies and lectins, targeting using "differential adsorption" [2] is a simpler - and therefore a more feasible - approach to site-specific drug delivery.

We wish to thank Mrs. Heike Betinski-Eggert for her technical assistance and Mr. Rüdiger Smal for preparing the photographs.

Received June 2, 1993

\section{References}

[1] Müller, R. H., Colloidal Carriers for Controlled Drug Delivery Modification. Characterization and in Vivo Distribution, CRC Press, Boca Raton 1991.

[2] Müller, R. H. and Heinemann, S., in: Gurny, R. and Junginger, H. E. (Eds.), Bioadhesion - Possibilities and Future Trends, Wissenschaftliche Verlagsgeselischaft, Stuttgart 1989, pp. 202-214.

[3] Brash, J. L., in: Brash, J. L. and Horbett, T. A (Eds.), Proteins at Interfaces: Physicochemical and Biochemical Studies, American Chemical Society, Washington D. C. 1987, pp. 490-506.

[4] Van Oss, C. J., Ann. Rev. Microbiol. 1978, 32, 19-39.

[5] Edebo, L. and Richardson, N., Int. Arch. Allergy Appl. Immunol. $1985,78,345-352$.

[6] Wilkins, D. J. and Myers, P. A., Br. J. Exp. Path. 1966, 47, 568-576.

[7] Schwendener, R. A., Lacocki, P. A. and Rahman, Y. E., Biochim. Biophys. Acta. 1984 772, 93-101.

[8] Norman, M. E., Williams, P., and Illum, L., Proceed, Intern. Symp. Control. Rel. Bioact. Mater. 1992, 19, 357-358.

[9] Hochstrasser, D. F., Harrington, M. G., Hochstrasser, A. C., Miller, M. J. and Merril, C. R., Anal. Biochem. 1988, 173, 424-435.

[10] Appel, R. D., Hochstrasser, D. F., Funk, M., Vargas, J. R., Pellegrini, C., Muller, A. F. and Scherrer, J. R., Electrophoresis 1991, 12, $722-735$.

[11] Illum. L., Davis, S. S., Müller, R. H., Mak, E. and West, P., Life Sci. $1987,40,367-374$.

[12] Illum. L. and Davis, S. S., Life Sci. 1987, 40, 1553-1560.

[13] Wesemeyer, H., Mikrokalorimetrische und chromatographische Untersuchungen zur Oberflächenmodifikation von partikulären Arzneistaffirägern durch Tenside, $\mathrm{Ph} . \mathrm{D}$. Thesis, University of Kiel, Germany 1993.

[14] Horbett, T. A., in: Winter, G. D., Gibbons, D. F., Plenk, H. (Eds.), Biomaterials 1980, Advances in Biomaterials, Wiley and Sons, Ltd, Chichester 1982, pp. 383.

[15] Horbett, T. A., in: Brash, J. L. and Horbett, T. A. (Eds.), Proteins at Interfaces: Physicochemical and Biochemical Studies, American Chemical Society, Washington D. C. 1987, pp. 239-260.

[16] Moghimi, S. M. and Patel, H. M., FEBS Lett. 1988, 233, 143-147.

[17] Moghimi, S. M. and Patel, H. M., Biochim. Biophys. Acta 1989, $984,379-383$.

[18] Moghimi, S. M. and Patel, H. M., Biochim. Biophys. Acta 1989, 984, 384-387.

[19] Fries, L. F. and Frank, M. M., in Stamatoyannopoulos, G., Nienhuis, A. W., Leder, P. and Majerus, P. W. (Eds.), The Molecular Basis of Blood Diseases, Saunders, Philadelphia 1987, pp. 450-485. 